\title{
In situ microstructure analysis of Inconel 625 during laser powder bed fusion
}

\author{
Felix Schmeiser $^{1, *}$ (D), Erwin Krohmer ${ }^{2}$, Christian Wagner ${ }^{1}$, Norbert Schell ${ }^{3}$, Eckart Uhlmann ${ }^{2,4}$, and \\ Walter Reimers ${ }^{1}$ \\ ${ }^{1}$ Institute for Materials Science and Technology, Metallic Materials, Technische Universität Berlin, Ernst Reuter Platz 1, 10587 Berlin, \\ Germany \\ ${ }^{2}$ Institute for Machine Tools and Factory Management (IWF), Technische Universität Berlin, Pascalstraße 8-9, 10587 Berlin, \\ Germany \\ ${ }^{3}$ Helmholtz-Zentrum Hereon, Max-Planck-Str. 1, 21502 Geesthacht, Germany \\ ${ }^{4}$ Fraunhofer Institute for Production Systems and Design Technology (IPK), Pascalstraße 8-9, 10587 Berlin, Germany
}

Received: 1 July 2021

Accepted: 29 September 2021

Published online:

8 November 2021

(C) The Author(s) 2021

\begin{abstract}
Laser powder bed fusion is an additive manufacturing process that employs highly focused laser radiation for selective melting of a metal powder bed. This process entails a complex heat flow and thermal management that results in characteristic, often highly textured microstructures, which lead to mechanical anisotropy. In this study, high-energy X-ray diffraction experiments were carried out to illuminate the formation and evolution of microstructural features during LPBF. The nickel-base alloy Inconel 625 was used for in situ experiments using a custom LPBF system designed for these investigations. The diffraction patterns yielded results regarding texture, lattice defects, recrystallization, and chemical segregation. A combination of high laser power and scanning speed results in a strong preferred crystallographic orientation, while low laser power and scanning speed showed no clear texture. The observation of a constant gauge volume revealed solid-state texture changes without remelting. They were related to in situ recrystallization processes caused by the repeated laser scanning. After recrystallization, the formation and growth of segregations were deduced from an increasing diffraction peak asymmetry and confirmed by ex situ scanning transmission electron microscopy.
\end{abstract}

Handling Editor: Sophie Primig.

Address correspondence to E-mail: felix.schmeiser@tu-berlin.de 


\section{GRAPHICAL ABSTRACT}

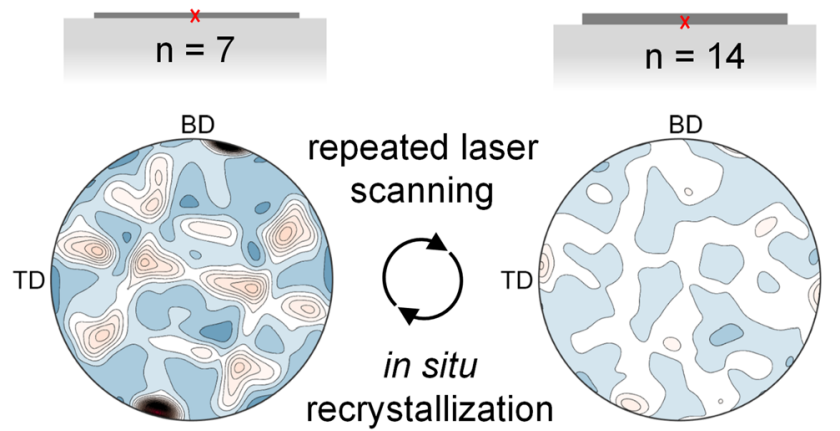

\section{Introduction}

Additive manufacturing facilitates the fabrication of arbitrarily complex geometries and tailored material properties using a range of different materials. Laser powder bed fusion (LPBF) is a process especially suited for the production of metal parts. LPBF employs focused laser radiation to melt a powder bed selectively. After laser exposure, the material solidifies immediately, and a new powder layer is applied, resulting in the layer-wise fabrication of the desired geometry. The selective and both locally and temporally varying energy input leads to a complex heat flow and temperature distribution, which govern the solidification and grain growth regime. Precise knowledge of the interaction of laser parameters and microstructural response within the workpiece opens up the prospect of tailored microstructure design.

Several properties characterize the microstructure of crystalline material, e.g., phase composition, microscopic defects such as porosity, and nanoscopic crystal lattice defects. Additionally, the grains' size, morphology, and crystallographic orientation severely impact the material's mechanical properties. The entirety of all crystallographic orientations in a polycrystalline material is summarized as its texture.

The texture is often described as the deviation from a random orientation distribution. The texture of a polycrystal is created during processes that define the microstructure, such as solidification and crystallization of a molten mass, recrystallization, and plastic deformation. The texture is determined by factors with a directed impact, such as mechanical force in plastic deformation or a directed heat flow during solidification [1].

A textured material exhibits anisotropic properties, e.g., Young's modulus, ductility, strength, and hardness. In LPBF, the directed solidification initially determines the texture as the material is exposed with a focused laser beam. The grain growth and the texture in a LPBF manufactured specimen are influenced by the melt pool geometry and the thermal gradients acting in the component [2]. As the melt pool solidifies, grains nucleate at the solidification front. During the subsequent grain growth, grains with different orientations compete [3]. Several studies found that grains with an orientation parallel to the melt pool boundary normal dominate during this phase $[2,4-6]$. This preferred orientation results from the heat flow, which has its largest magnitude in the melt pool boundary's normal direction [7]. The preferred orientation is, furthermore, dependent on the crystal lattice. For example, face-centered cubic (fcc) metals such as the nickel-base alloy Inconel 625 grow in the preferred $\langle 100\rangle$ direction [1].

The melt pool geometry depends on several factors, e.g., thermal conductivity, energy absorption, and heat flow [6, 8]. Gong et al. [9] investigated the influence of different laser powers and scanning speeds on the melt pool geometry. They found that high laser powers and low scanning speeds lead to the keyhole effect. Here, the melt pool shows a broad opening at the top and tapers toward the bottom. The keyhole has a high penetration depth due to the high laser intensity and energy input, resulting in the remelting of previously solidified material. 
Furthermore, keyholing leads to undesirable porosity [10]. Contrarily, a combination of low laser power and high scanning speeds leads to the balling effect. If the energy input is insufficient, the layer below will be insufficiently wetted. Spherical melt pools and bead-like structures are formed, which impede the following layers and can disrupt the manufacturing process by jamming the powder recoating mechanism [11].

The energy input also impacts grain growth. Higher laser powers lead to deeper melt pools, lower laser powers to shallower melt pools. In shallower melt pools, the normal vectors on the melt pool boundaries are more parallel than in deeper melt pools. They are parallel to the building direction of the part, therefore resulting in a preferred orientation in the building direction [6]. The melt pools are deeper when using lower scanning speeds or high laser powers, leading to a more significant variation of the melt pool boundary normal directions [4]. Therefore, crystallites do not only grow in the building direction, resulting in a lower preferred orientation [6]. Sun et al. [4] concluded that texture development could be controlled via process control. They successfully built parts from 316L, which showed a $\langle 011\rangle$ fiber texture instead of the commonly found $\langle 001\rangle$ for fcc alloys by using an increased laser power and a multi-scan method.

Yin et al. [5] found a dependence between the orientation of grain growth and laser power and scanning speed using FEM simulations for Ti-6Al-V4 processed via LPBF. They concluded that the layer number exerts an influence on the melt pool geometry and, therefore, on the grain orientation. For small layer numbers, the substrate plate strongly influences heat dissipation. Here, the crystal orientations show substantial deviations from the building direction. With increasing layer numbers, the melt pool becomes shallower, and the grain growth changes toward the building direction after a few layers. [5]

Several approaches to tailor the microstructure via process parameter manipulation have been published to date. Roehling et al. [12] adjusted the shape of the laser beam to generate preferable microstructures and mechanical properties. Dehoff et al. [13] showed that tailored, site-specific textures could be designed by varying the process parameters. The typical route is a post-process heat treatment, usually aimed at achieving a recrystallized microstructure [14]. For Inconel 625, Marchese et al. [15] investigated the microstructural evolution after various heat treatments and found a recrystallized microstructure after a solution heat treatment. Sabzi et al. [16] recently presented the first experimental proof that dynamic recrystallization (DRX) occurs during LPBF as well, using ex situ EBSD measurements and thermomechanical modeling. However, an in situ observation of DRX during LPBF has not been reported yet.

Since DRX impacts the crystallographic texture, high-energy synchrotron radiation diffraction is wellsuited for its investigation. In situ diffraction experiments so far have been focused on phase transformations [17], cooling dynamics [18, 19], and internal stresses [20, 21]. Recently, Wahlmann et al. [22] presented results regarding the in situ formation of precipitates, another important constituent of the microstructure, in a nickel-base superalloy using synchrotron radiation diffraction and the LPBF device from the present study's group.

To the authors' knowledge, for LPBF, in situ texture analyses using high-energy synchrotron radiation have not been reported so far. Using a custombuilt LPBF machine designed for in situ diffraction measurements, experiments using Inconel 625 were carried out to determine the development of texture and the influence of repeated laser scanning on the microstructure.

\section{Materials and methods}

\section{Custom LPBF machine}

In previous work, a custom LPBF process chamber was developed and integrated into the modular LPBF machine AconityMINI from Aconity3D GmbH, Herzogenrath, Germany, to realize in situ X-ray diffraction experiments with synchrotron radiation [23]. The custom LPBF machine is equipped with a $400 \mathrm{~W}$ Ytterbium fiber laser YLR-400-AC from IPG Laser $\mathrm{GmbH}$, Burbach, Germany. The laser source emits light with a wavelength of $1070 \mathrm{~nm}$ in continuous wave. The laser is focused and deflected on the powder bed with an Axialscan-30 from Raylase $\mathrm{GmbH}$, Wessling, Germany, with a focus diameter of ca. $60 \mu \mathrm{m}$ in $1 / \mathrm{e}^{2}$ specification. Before processing, the process chamber was sealed and purged with argon. During the processing, a clean argon atmosphere was maintained by using a filtration unit connected to the process chamber and 
coupled to a circulation pump. The powder bed was sandwiched between two glassy carbon windows. Inlet and outlet windows for the synchrotron radiation in the process chamber are made from polyimide foil. An automatic powder recoating mechanism inside the purged process chamber ensured the additive manufacturing of multi-layer parts without interrupting the experiment for manual operations.

\section{Materials}

The nickel-base alloy 625, commonly referred to as Inconel 625, was used as powder material and purchased from $\mathrm{m} 4 \mathrm{p}$ material solutions $\mathrm{GmbH}$, Magdeburg, Germany. The particles were nitrogenatomized and exhibited mostly spherical shapes, Fig. 1, with particle sizes ranging from $20 \mu \mathrm{m}$ to $63 \mu \mathrm{m}$, Table 1 . The median circularity of the

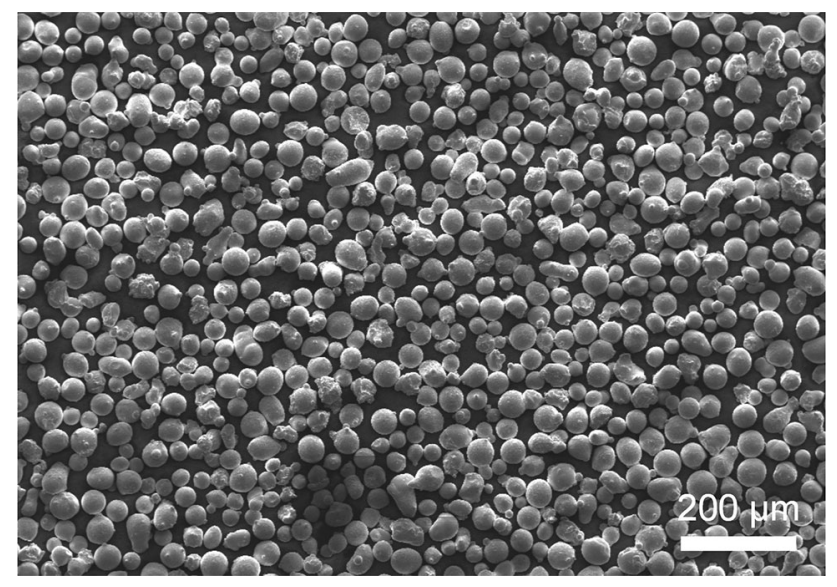

Figure 1 SEM image of Inconel 625 powder.

Table 1 Particle size distribution of Inconel 625 powder per sieve analysis

\begin{tabular}{lllll}
\hline $0-20 \mu \mathrm{m}$ & $20-40 \mu \mathrm{m}$ & $40-50 \mu \mathrm{m}$ & $50-63 \mu \mathrm{m}$ & $+63 \mu \mathrm{m}$ \\
\hline $0.68 \%$ & $67.64 \%$ & $29.42 \%$ & $2.26 \%$ & $0.0 \%$ \\
\hline
\end{tabular}

particles, calculated with Eq. 1, was 0.85 with circ $=1$ equaling a perfect circle.

circ $=4 \pi \times \frac{\text { Area }}{\text { Perimeter }^{2}}$,

Structural steel S235 plates with a size of $70 \times$ $20 \times 3 \mathrm{~mm}^{3}$ were used as substrate material.

\section{Process parameters and measurement modes}

Two different LPBF parameter sets were investigated, Table 2. They are composed of two pairs of laser power $P_{\mathrm{L}}$ and scanning speed $v_{\mathrm{L}}$ ranging from a low laser power $P_{\mathrm{L}}=55 \mathrm{~W}$ and low scanning speed $v_{\mathrm{L}}=50 \mathrm{~mm} / \mathrm{s}$ to a high laser power $P_{\mathrm{L}}=275 \mathrm{~W}$ and a high scanning speed $v_{\mathrm{L}}=760 \mathrm{~mm} / \mathrm{s}$. The ratio of laser power $P_{\mathrm{L}}$ to scanning speed $v_{\mathrm{L}}$ is denoted as line energy $E_{\mathrm{L}}$. The scanning pattern featured unidirectional scanning vectors aligned longitudinally to the incident synchrotron radiation beam, Fig. 2 . Cuboid samples ca. $20 \times 2.5 \times 5 \mathrm{~mm}^{3}$ in size were produced out of 100 layers with a layer thickness of $\Delta \mathrm{z}=50 \mu \mathrm{m}$. Laser scanning was set to start at the left edge and finish at the right edge of the sample geometry. Two different measurement modes were used. In measurement mode 1 (MM1), the gauge volume (GV) distance to the working plane, $z_{\mathrm{GV}}$, is kept constant throughout the measurement of all the 100 layers. In measurement mode 2 (MM2), the absolute gauge volume position in the sample is maintained such that a defined volume element of the sample, defined by its distance to the samplesubstrate interface, $\zeta_{\mathrm{GV}}$, is tracked throughout the process.

In this study, the axis parallel to the incident synchrotron radiation beam is called longitudinal direction (LD), which spans the working plane together with the transverse direction (TD). The third axis is parallel to the part height and called the building direction (BD).

Table 2 Parameter sets for the experiments

\begin{tabular}{lllllll}
\hline $\begin{array}{l}\text { Parameter } \\
\text { unit }\end{array}$ & $\begin{array}{l}\text { Laser power } P_{\mathrm{L}} \\
{[\mathrm{W}]}\end{array}$ & $\begin{array}{l}\text { Scanning speed } v_{\mathrm{L}} \\
{[\mathrm{mm} / \mathrm{s}]}\end{array}$ & $\begin{array}{l}\text { Line energy } E_{\mathrm{L}} \\
{[\mathrm{J} / \mathrm{mm}]}\end{array}$ & $\begin{array}{l}\text { Scanning pattern } \\
{[\mu \mathrm{m}]}\end{array}$ & $\begin{array}{l}\text { Hatch distance } h_{\mathrm{L}} \\
{[\mu \mathrm{m}]}\end{array}$ & $\begin{array}{l}\text { Layer thickness } \Delta \mathrm{z} \\
\text { Set } 1\end{array}$ \\
\hline Set 2 & 275 & 50 & 1.1 & Longitudinal & 120 & 50 \\
\hline
\end{tabular}


Figure 2 Experimental procedure: a Measurement modes and gauge volume positioning; b Laser scanning pattern. (a) Measurement mode 1:

Fixed distance to working plane $\mathrm{z}_{\mathrm{GV}}$

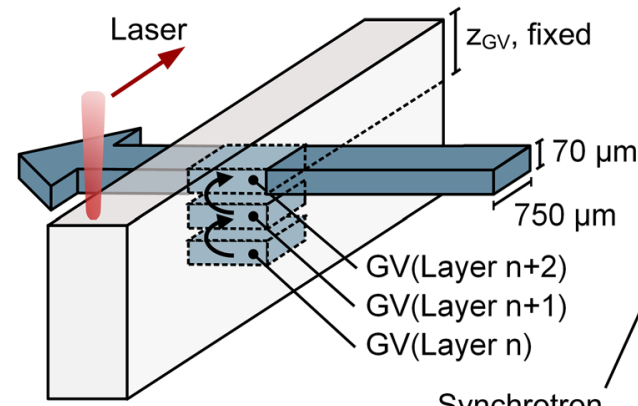

Synchrotron

(b)

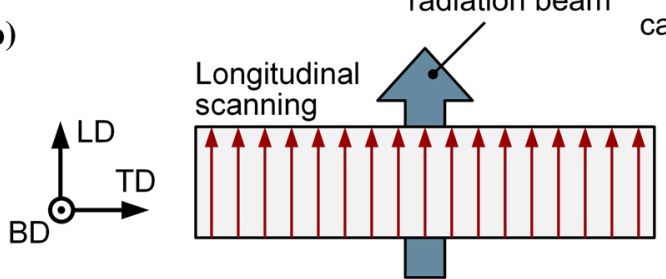

Measurement mode 2:

Fixed absolute position in $B D \zeta_{G V}$

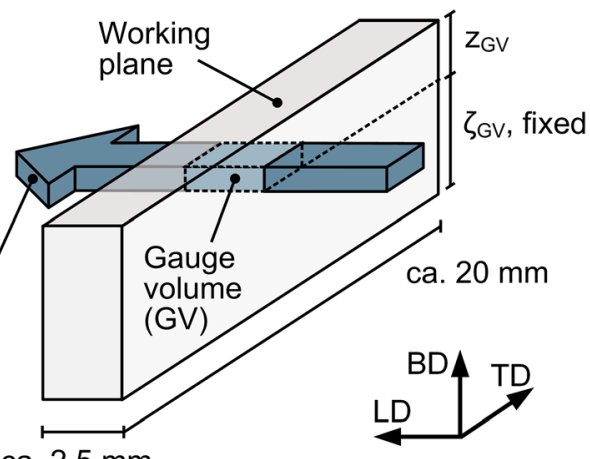

\section{Experimental procedure}

In situ diffraction experiments were carried out at PETRA III, Deutsches Elektronen-Synchrotron (DESY) in Hamburg, Germany. The High Energy Materials Science (HEMS) beamline P07 [24], which is operated by Helmholtz-Zentrum Hereon, features a heavy load 6-axis positioning system on which the process chamber of the custom LPBF machine was mounted. A PerkinElmer XRD1621 area detector was used for diffraction pattern acquisition. For the diffraction experiments, synchrotron radiation energies of 87 and $98 \mathrm{keV}$ were used. The synchrotron radiation beam size was set to $750 \mu \mathrm{m}$ in TD and $70 \mu \mathrm{m}$ in BD, Fig. 2.

The diffraction patterns were acquired with an exposure time $\mathrm{t}=0.1 \mathrm{~s}$ and frequency $\mathrm{f}=10 \mathrm{~Hz}$. Patterns were collected as layer-wise image series when the laser emission was active. The series measurement started with the laser being turned on and ended at the end of laser scanning. Therefore, not the whole cooling process after the laser impact was observed.

To complement the in situ diffraction experiments, two specimens were analyzed after production using standard metallographic preparation methods. For both parameter sets, the TD-BD plane was polished and etched in a solution of $1.5 \mathrm{ml}$ hydrogen peroxide and $50 \mathrm{ml}$ hydrochloric acid for $\mathrm{t}=45 \mathrm{~s}$. This preparation emphasized grain structures and melt pool boundaries in the subsequent optical microscopy using a Zeiss Axioskop 50.

Additionally, one sample, manufactured using $\mathrm{P}_{\mathrm{L}}=55 \mathrm{~W}$, was investigated by scanning transmission electron microscopy (STEM) at Zentraleinrichtung Elektronenmikroskopie (ZELMI) of Technische Universität Berlin. The specimen was cut using a focused ion beam on a FEI Helios Nanolab 600 FIB to ensure that the subsequent STEM analysis probed the same gauge volume as the in situ diffraction experiments in MM2. The investigations were carried out using a JEOL JEM-ARM300F2 STEM with a cold field emission gun and a probe-Cs corrector operating at an acceleration voltage of $300 \mathrm{kV}$ and equipped with a JEOL Dual-EDX system using two $160 \mathrm{~mm}^{2}$ SDD detectors. High-angular annular dark-field images (HAADF) were acquired because of their high sensitivity to the atomic number of the probed material.

\section{Data evaluation}

The detector calibration and integration were performed using the Python library pyFAI [25], pole figures were generated using MTEX [26]. The detector calibration was performed using a standard LaB6 powder sample to determine the detector-sample distance and the detector tilt for accurate integration results. Subsequently, the diffraction patterns were integrated in $5^{\circ}$ sectors, resulting in 72 line profiles, including the first five hkl reflections for each 

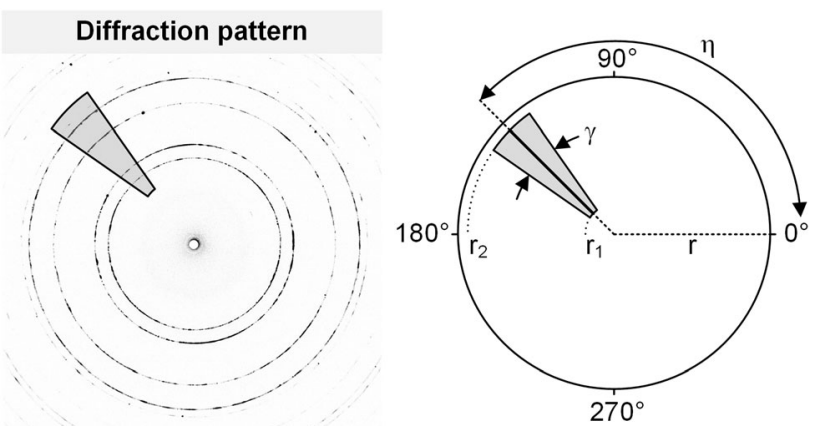

Integration Parameters

$\eta \in\left[0^{\circ} ; 5^{\circ} ; 10^{\circ} ; \ldots ; 355^{\circ}\right] \quad$ Azimuth angle

$\gamma=5^{\circ}$

Opening angle
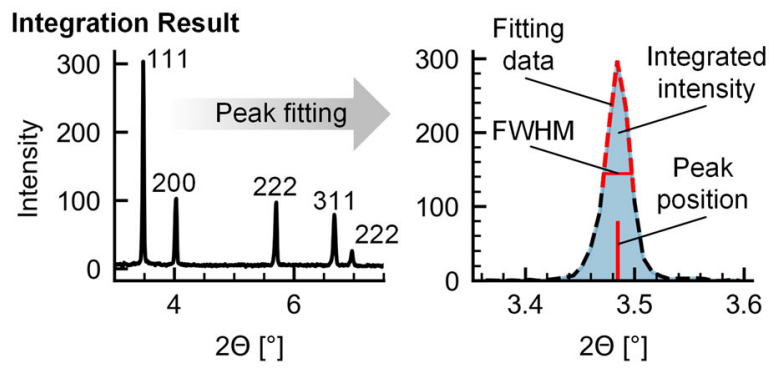

Figure 3 Data evaluation procedure from sector integration to peak fitting.

diffraction pattern, Fig. 3. For each reflection, background subtraction was performed. Then, the integrated intensity was calculated following the trapezoid rule.

Additionally, the peak position was determined by fitting the line profile with a PseudoVoigt function using the python library LMFIT [27]. This fitting function also yielded the full width at half maximum (FWHM) of the reflections. For the fitting, only the top $60 \%$ of each peak were considered due to a slight asymmetry of the profiles, further discussed in Sect. 3.3. The peak positions and integrated intensities were converted to a format readable by MTEX to calculate the orientation distribution function (ODF), which was then plotted as a pole figure. This procedure was derived from Wenk and Grigull [28].

Representative results gathered from in situ experiments illustrate the evolution of microstructural features during LPBF. The data is presented based on two visualization styles. Pole figures represent the standard method of conveying texture. They show a color-coded intensity distribution as multiples of a random distribution (MRD). Each pole figure is calculated considering both the intensity and symmetry of the first five hkl reflections. The colormap chosen for plotting the pole figures emphasizes deviations from a random distribution, which would equal no preferred orientation. In this work, pole figures are used to visualize single texture states.

Temporally resolved texture evolution is visualized by time-dependent integrated intensity distributions, which are called processing plots in this study. These distributions show the azimuthal intensity for the (111) and (200) reflections. This way, local changes in the intensity distribution over time are visible. These visualizations do not contain the same amount of information as pole figure plots, though, which is why they are used complementarily.

\section{Results}

\section{Impact of laser irradiation on texture}

In Fig. 4, the impact of the laser scanning over the gauge volume is shown for two different energy inputs, denoted by the different laser powers $P_{\mathrm{L}}$. For both laser powers, the changes in texture are documented for a single layer. Pole figures are given for the initial state, before laser impact, and the final state, at the end of the laser scanning time. Additionally, time-resolved intensity changes are made visible through the processing plots. Here, azimuths of $0^{\circ}, 180^{\circ}$, and $360^{\circ}$ correspond to TD, while $90^{\circ}$ and $270^{\circ}$ correspond to $\mathrm{BD}$. In both measurements, the gauge volume was positioned at $z_{\mathrm{GV}}=150 \mu \mathrm{m}$ below the top surface of the part. The azimuthal intensities for the (111) and (200) reflections were normalized for each sample since the respective experiments were conducted with varying incident beam intensities and energies.

The $275 \mathrm{~W}$ sample showed an intensification of the orientation distribution after the laser has passed over the gauge volume. The same parallel lines persist, with the intensities being much higher after the laser peak. The pole figures confirm this observation. The (200) pole figure shows an intensification of texture as the maximum MRD value increases. The local intensity maxima around $\mathrm{BD}$ are much more pronounced in the final state than the initial state. Similar textures were found in other build heights as well, Fig. 5, showing that this parameter set leads to a homogeneous texture in the part. 
(111)
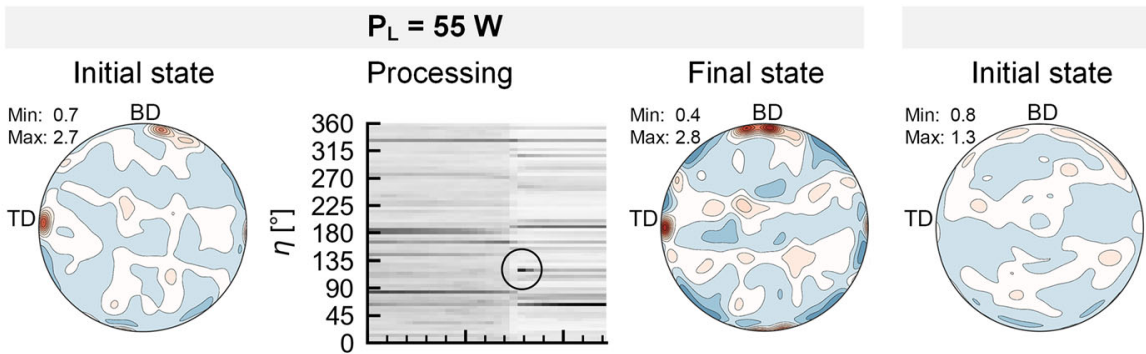

$$
P_{\mathrm{L}}=275 \mathrm{~W}
$$
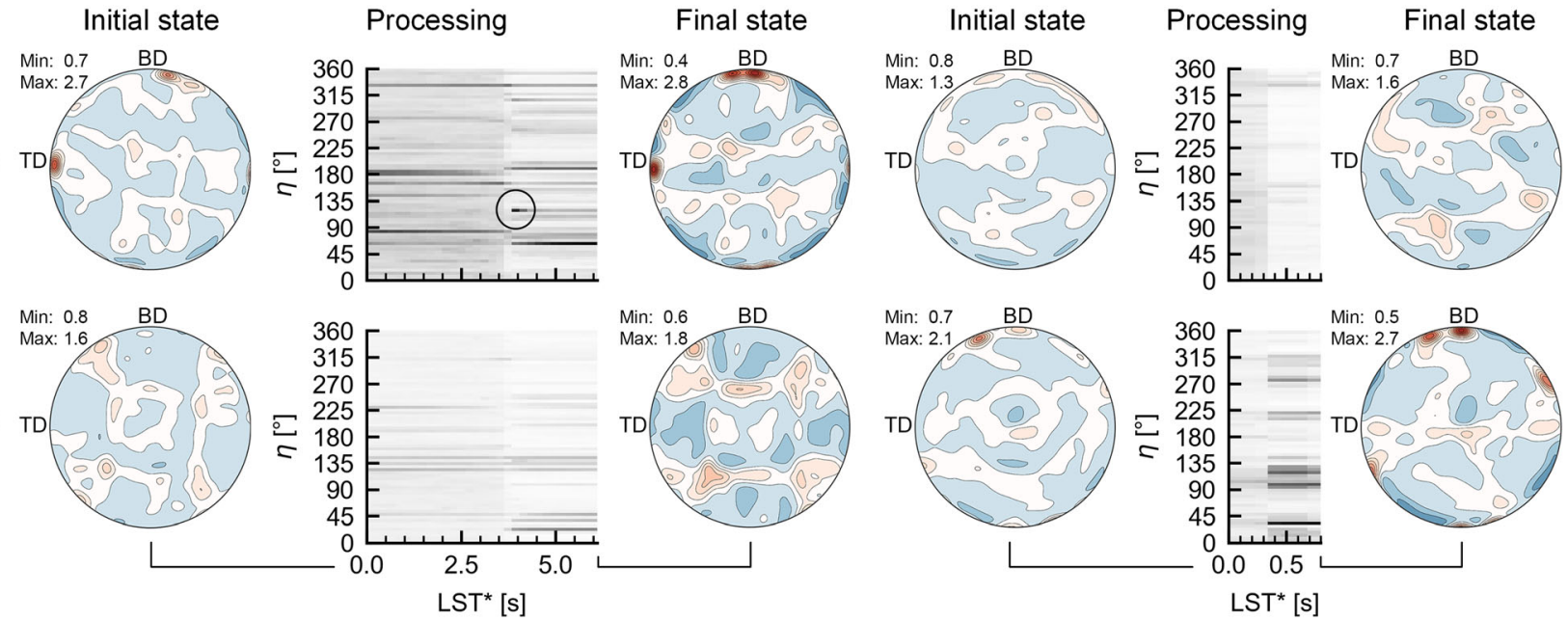

(200)

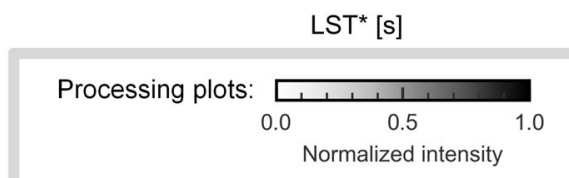

Pole figure plots:
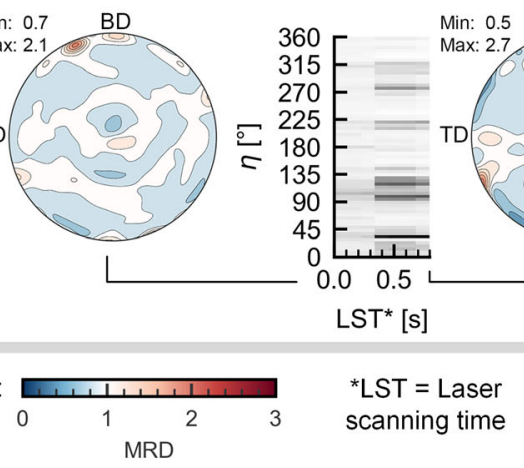

Figure 4 Comparison of the influence of different energy inputs on texture: Intensity distribution throughout a single layer in MM1 for $\mathrm{n}=50, z_{\mathrm{GV}}=150 \mu \mathrm{m}$. Circled area in processing plot for $P_{\mathrm{L}}=55 \mathrm{~W}$ shows high intensity that rapidly decreases after laser impact.

The $55 \mathrm{~W}$ sample, on the other hand, shows a different orientation distribution after the laser impact compared to before. Initially, the highest intensities were found for the (111) reflection in both TD and $\mathrm{BD}$. As the laser scans over the gauge volume, a highintensity spot appears at $120^{\circ}$, which fades quickly. The intensity line in $\mathrm{BD}$ shifts toward $45^{\circ}$, while the one at $180^{\circ}$ is shifted to a larger azimuth angle. The (200) reflection shows lower intensities than the (111) reflection with less distinctive features, except for intensity lines emerging between $20^{\circ}$ and $45^{\circ}$ after laser impact. The (200) pole figures change drastically from the initial to the final state, reflecting the (200) reflection's response to a (111) fiber texture. In the (111) pole figure, a spot at TD is visible in both the initial and final states, while a slightly inclined spot from BD appears to shift counterclockwise in the final state.

In Fig. 6, optical micrographs of the part-substrate interface regions of both samples are shown. This section was chosen because of the clarity of the melt pool boundaries. The low laser power and high line energy result in clearly deeper melt pools than the higher laser power, which features broader and shallower melt pools. The higher laser power shows columnar grains parallel to $\mathrm{BD}$, which surpass the

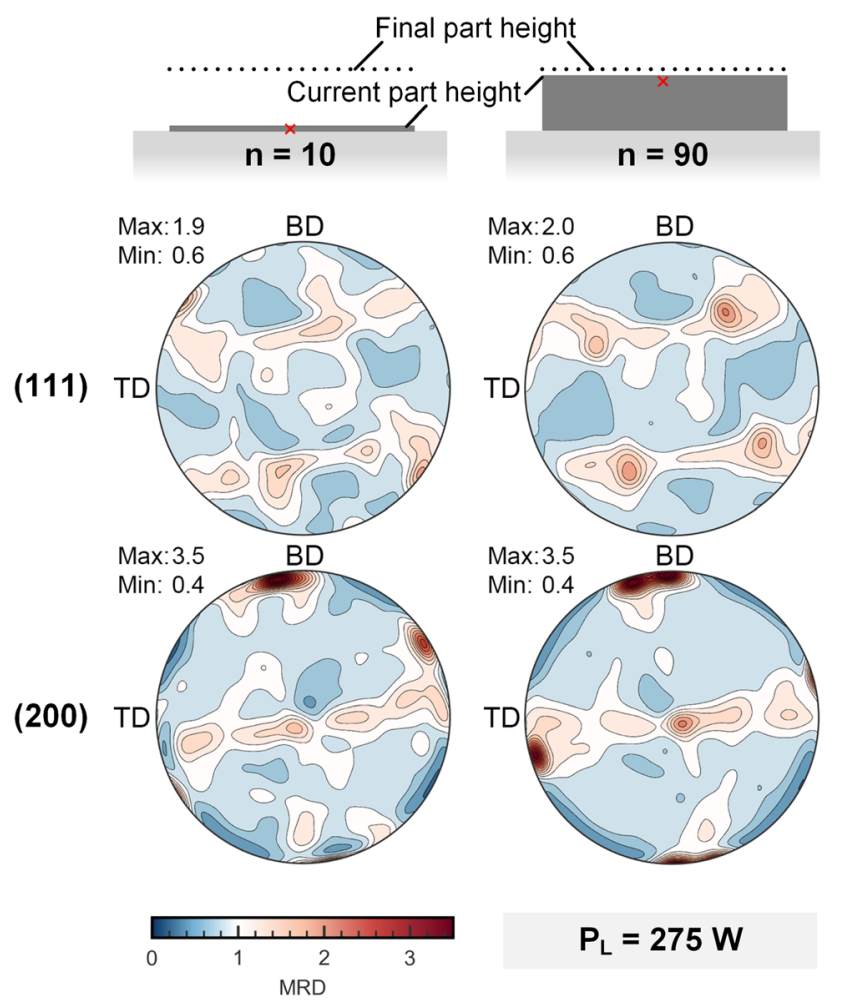

Figure 5 Final state texture in different build heights within a sample manufactured with a laser power of $P_{\mathrm{L}}=275 \mathrm{~W}$ and observed in MM1 at $z_{\mathrm{GV}}=150 \mu \mathrm{m}$. 
Figure 6 Ex situ micrographs of the interface region of part and substrate for both parameter sets.
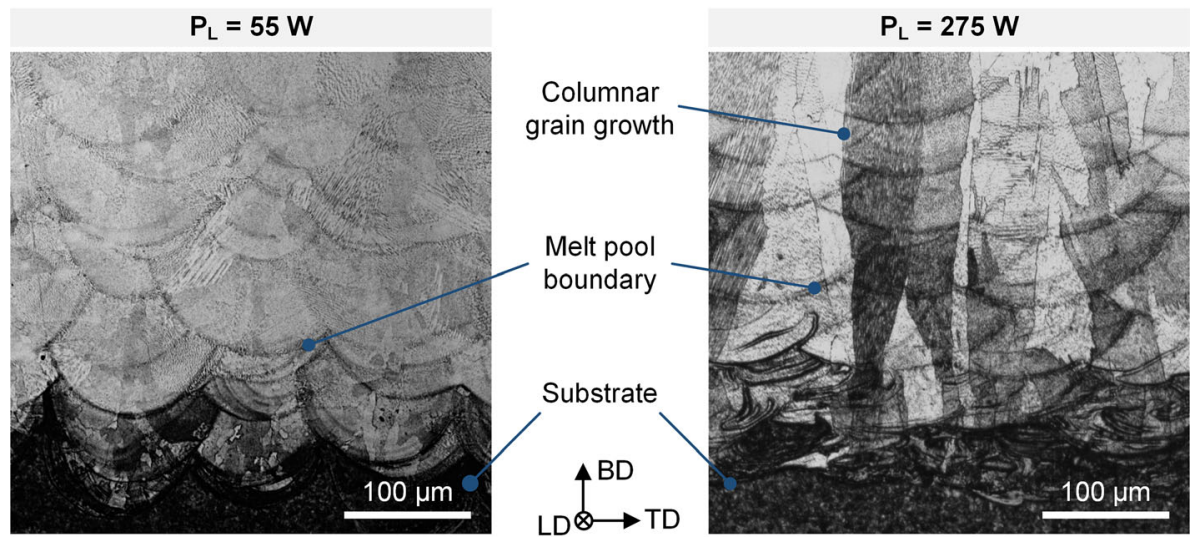

(111)
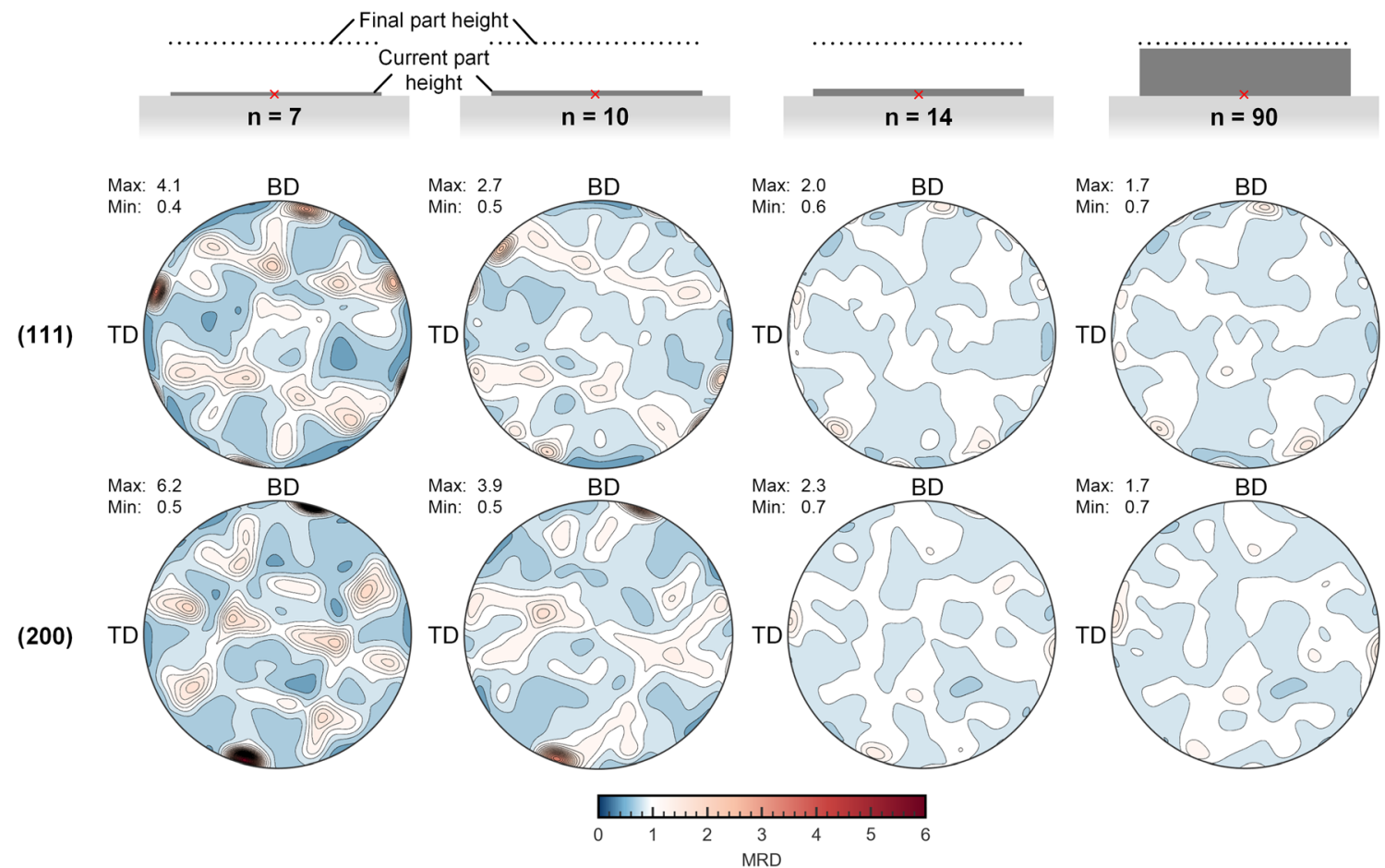

Figure 7 Texture evolution with repeated laser passes over the gauge volume within a sample manufactured with a laser power of $\mathrm{P}_{\mathrm{L}}=55 \mathrm{~W}$ and observed in MM2 at $\zeta_{\mathrm{GV}}=250 \mu \mathrm{m}$.

melt pool boundaries. The grains visible in the micrographs are larger for the high laser power than for the low laser power, where the grain morphology appears less oriented.

\section{Impact of repeated laser scans on texture}

Another phenomenon appears when observing a constant gauge volume throughout the whole process. Figure 7 shows pole figures of the same gauge volume at increasing numbers of total layers. Each pole figure represents the final state after laser scanning, similar to Fig. 4 . The gauge volume was located in the center of the fifth layer of the sample at $\zeta_{\mathrm{GV}}=250 \mu \mathrm{m}$. For each pole figure set, the total number of layers is given below the estimated total part height at that moment. For $n=7$, consequently, two additional layers of solidified material were above the gauge volume, five layers for $n=10$ et cetera.

Initially, a bimodal fiber texture is observed at $n=7$. The (200) plane shows a strong preferred 
Figure 8 Sample

manufactured with a laser power of $\mathrm{P}_{\mathrm{L}}=55 \mathrm{~W}$ and observed in MM2 at $\zeta_{\mathrm{GV}}=250 \mu \mathrm{m}$ : Processing plots for (111) and (200) reflections.
(111)

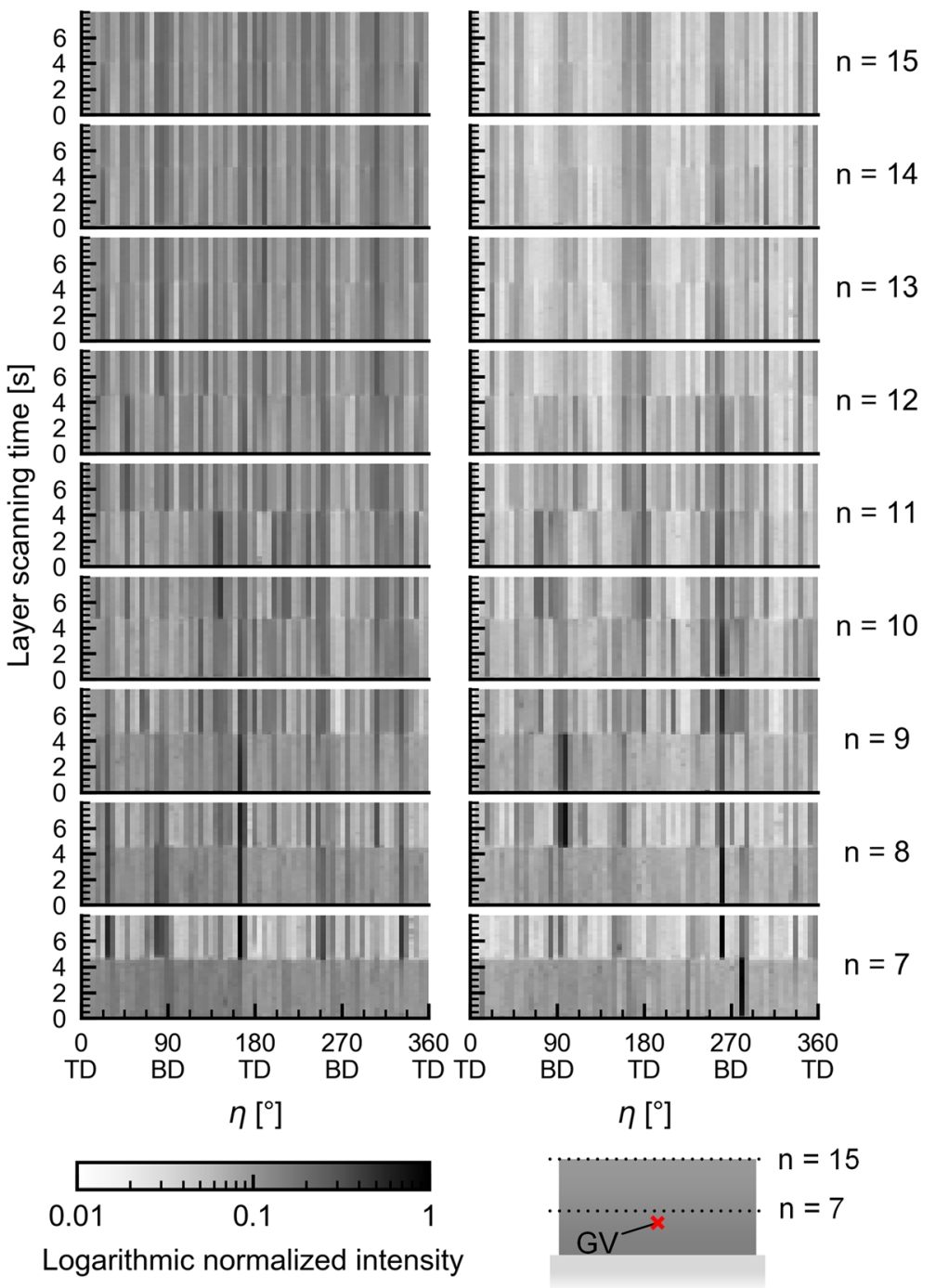

orientation in building direction with a slight inclination. Lower intensity ribbons along the TD plane indicate a (200) fiber texture. Simultaneously, the (111) pole figure shows a fiber texture, too, with the fiber axis slightly tilted from the equatorial plane TD.

At $\mathrm{n}=10$, five layers have been exposed atop the gauge volume, equaling five additional laser passages and about $250 \mu \mathrm{m}$ of solidified material above. Here, the (111) fiber texture seems to have almost completely vanished, while the (200) fiber texture is still visible and pronounced. Still, the maximum intensity is significantly reduced compared to $n=7$. At $\mathrm{n}=14$, the intensities are further reduced. The (111) pole figure has completely changed compared to its initial state at $\mathrm{n}=7$, and the fiber texture for the (200) plane is further weakened, signified by a maximum intensity of only 2.4 compared to 6.2 for the first pole figure.

In Fig. 8, the changes in the intensity distributions are temporally resolved. The azimuthal intensity distribution for the (111) and (200) reflections are shown layer by layer from $n=7$ to $n=15$. A logarithmic intensity scale was used to unveil all intensity changes in an extensive range for this figure.

The laser passage over the gauge volume is visible in each plot, signified by a slight intensity change at about $t=4 \mathrm{~s}$. As more material is deposited above the gauge volume, this effect gets weaker. At $n=7$, the initial (111) texture seen in the corresponding pole figure in Fig. 7 is created. Before the laser impact, the intensity distribution appears homogeneous. Afterward, five significant lines emerge, 
(a)

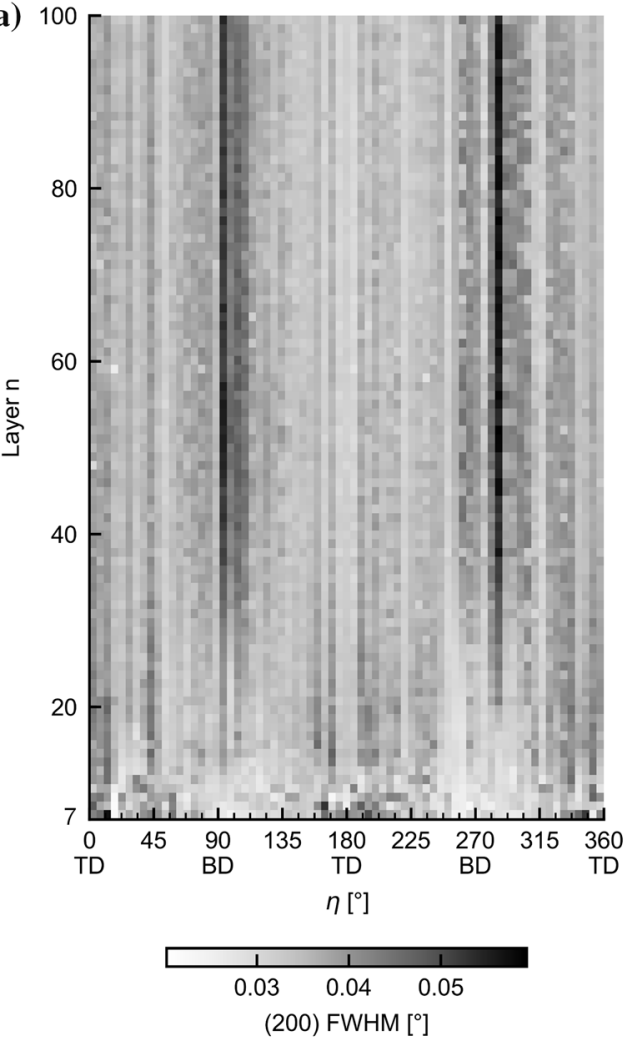

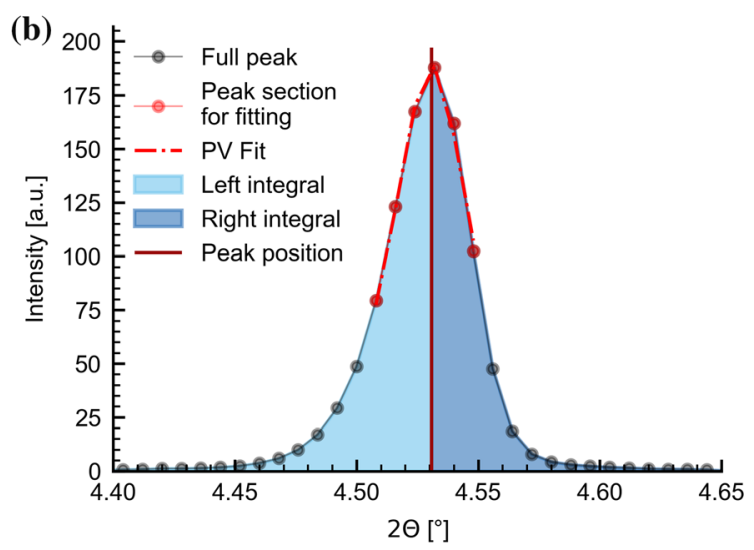

(c)

$(111)$

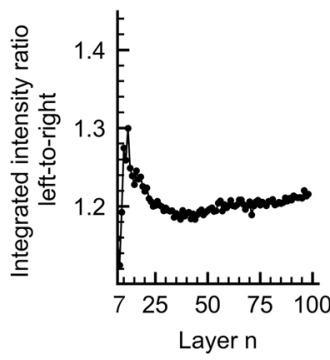

(200)

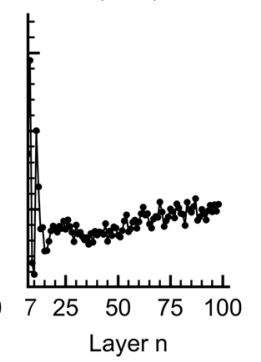

(220)

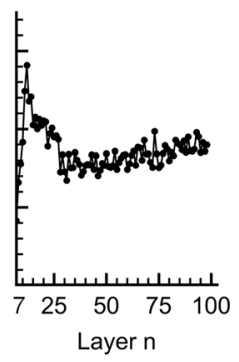

Figure 9 Sample manufactured with a laser power of $\mathrm{P}_{\mathrm{L}}=55 \mathrm{~W}$ and observed in MM2 at $\zeta_{\mathrm{GV}}=250 \mu \mathrm{m}$ : a Azimuthal (200) FWHM distribution at the end of each layer; $\mathbf{b}$ Peak shape analysis and fitting parameters; $\mathbf{c}$ Peak skewness parameter for first three hkl reflections.

which persist until the laser impact at $\mathrm{n}=9$. The (200) reflection shows changes in the intensity distribution as well. Initially, there is a slight shift of a prominent intensity at $270^{\circ}$ to about $255^{\circ}$. This line persists up to $\mathrm{n}=15$ and further onwards. After $\mathrm{n}=14$, no further changes in either intensity distribution were observed, which mirrors the findings from the pole figures in Fig. 7.

\section{Repeated laser scanning distorts peak shape}

Further analysis of the peak shape was carried out to evaluate possible solid-state phenomena occurring during LPBF. Initially, the FWHM was determined as described in Sect. 2.5, as shown in Fig. 9b.

The azimuthally resolved results are shown in Fig. 9a. Each horizontal line corresponds to the azimuthal distribution of the (200) reflection's FWHM at the end of the corresponding layer. Up to layer $\mathrm{n}=10$, the image appears relatively undefined with changing distributions layer by layer. At this stage, the LPBF process has likely not reached a steady-state concerning the deposited and solidified layer thickness [29]. Generally, the FWHM values appear higher for TD than for BD in this region, though.

After $\mathrm{n}=20$, two prominent lines emerge at around $90^{\circ}$ and $270^{\circ}$, corresponding to increased peak widths in the building direction. Finally, between $n=30$ and $n=40$, these lines reach their maximum, with the rest of the distribution not changing anymore. This increase was further evaluated since the amount of material of about 1.25 to $1.5 \mathrm{~mm}$ between the gauge volume and the laserimpacted powder layer seemed significant, and the intensity distributions shown in the previous chapter stayed unchanged at these layer numbers.

The peak shape was determined to be one possible factor to distort the FWHM value. The measured peak profiles are slightly asymmetrical with a less steep left side. If this asymmetry were to increase over time, it would impact the FWHM value. The integrated intensity was divided into two sections to evaluate the asymmetry: left of the peak position and right of the peak position. As mentioned before, the peak position was determined via a PseudoVoigt fit of the top $60 \%$ of the peak, where the impact of

\section{Springer}




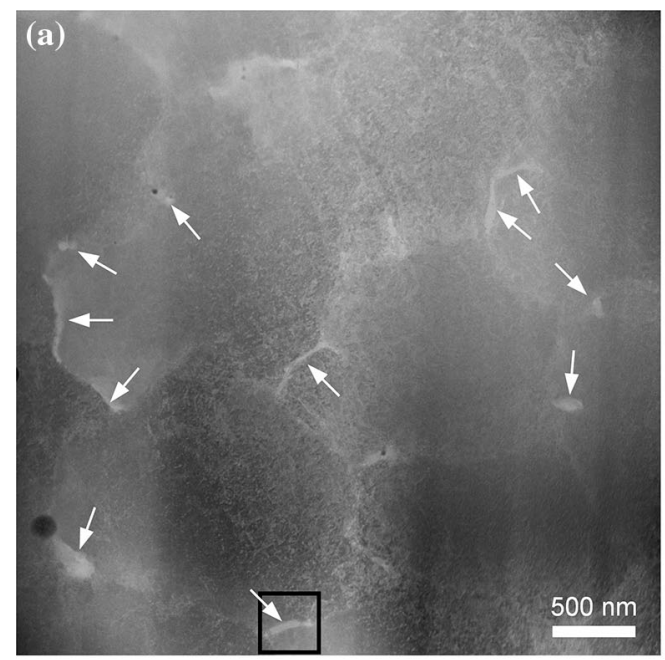

Figure 10 High-angular annular dark-field images acquired by STEM with brighter areas corresponding to higher atomic numbers: a Overview image where white arrows indicate $\mathrm{Nb}$

asymmetry is negligible. Therefore, the left and rightside integrals were calculated separately. The ratio of left-to-right was used as an asymmetry factor. Its evolution over time is shown in Fig. 9c for the first three hkl reflections (111), (200), and (220). The remaining two collected reflections (311) and (222) showed similar behavior.

After an initial fluctuation, the ratio reaches a local minimum between $\mathrm{n}=20$ and $\mathrm{n}=25$ depending on the reflection, after which it slowly increases linearly. Therefore, the peaks become more asymmetrical over time, all while the material stays in a solid state, and the impact of the laser on the gauge volume is expected to be reduced as the amount of material between gauge volume and top layer increases. It was therefore concluded that the asymmetry was not the deciding factor behind the FWHM increase described above.

\section{Ex situ microstructure analysis}

The influence of repeated laser scanning on the microstructure was further evaluated using highresolution scanning transmission electron microscopy (STEM). The sample using $P_{\mathrm{L}}=55 \mathrm{~W}$ observed in MM2 was investigated in the region where the synchrotron gauge volume was located. A high-angular annular dark-field image detector was used due to its high sensitivity to the atomic number and the resulting $\mathrm{Z}$ contrast. Atoms with a higher atomic number appear brighter in the resulting image. The

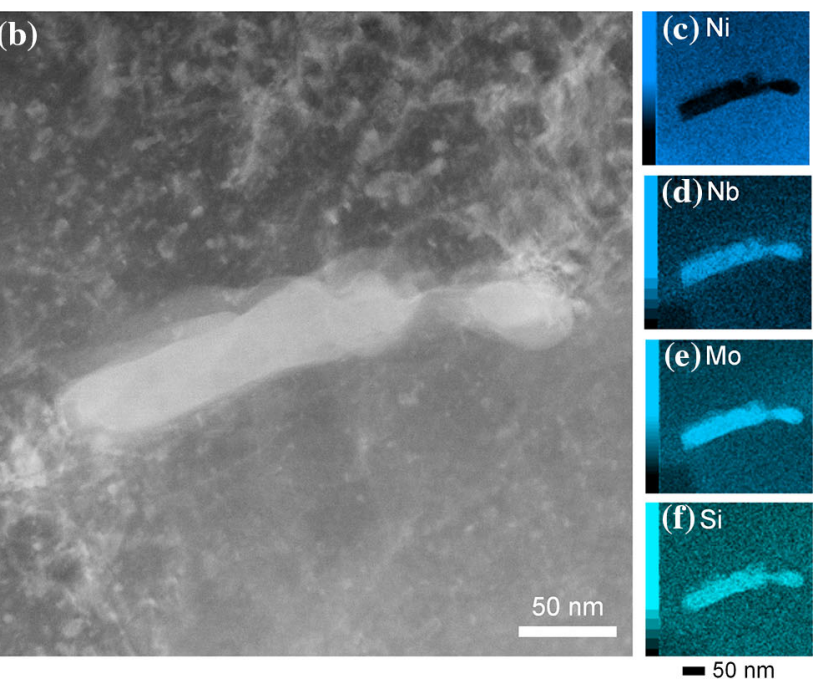

and Mo-rich areas; $\mathbf{b}$ Close-up of framed area in overview image; c-f STEM-EDX maps for nickel, niobium, molybdenum, and silicon.

overview in Fig. 10a shows an inhomogeneous microstructure with a lot of contrast. Several bright features with a size of $100-300 \mathrm{~nm}$ in the interdendritic regions stand out. Energy-dispersive X-ray spectroscopy (EDX) images in Fig. 10c-f reveal that these segregations have high $\mathrm{Nb}$ and $\mathrm{Mo}$ as well as $\mathrm{Si}$ contents.

\section{Discussion}

\section{Influence of energy input}

As described in the introduction, the energy input is one of the deciding factors for forming preferred orientations in the material. In this study, the parameter set with the lower laser power of $P_{\mathrm{L}}=55 \mathrm{~W}$ had the higher energy input with a line energy of $E_{\mathrm{L}}=1.1 \mathrm{~J} / \mathrm{mm}$ compared to a line energy of $E_{\mathrm{L}}=0.36 \mathrm{~J} / \mathrm{mm}$ for the parameter set with the higher laser power of $P_{\mathrm{L}}=275 \mathrm{~W}$. Generally, higher energy inputs result in deeper melt pools and less textured material, which was confirmed in the present study [4].

The high laser power parameter set showed a (200) fiber texture along the building direction regardless of build height and layer number, while the low laser power did not result in a significant preferred orientation. The parameter set with a line energy of $E_{\mathrm{L}}=0.36 \mathrm{~J} / \mathrm{mm}$ resulted in shallower melt pools, therefore, more parallel grain growth directions. As a 
result, crystallites grow epitaxially, signified by the intensification of existing orientations and the columnar grain growth transcending the melt pool boundaries.

The parameter set with a line energy of $E_{\mathrm{L}}=1.1 \mathrm{~J} /$ $\mathrm{mm}$ leads to deeper melt pools and a reduced preferred orientation. The changes in the intensity distribution during laser scanning imply a certain depth of the heat-affected zone that enables orientation changes. The single intensity spot visible only for a short period during laser scanning, annotated in Fig. 4, indicates the consumption of grains during grain growth.

\section{In situ recrystallization stage}

The measurements in a constant gauge volume revealed significant changes in the intensity distribution in solidified material, which coincide with a reduction in texture. The texture changes from a strong bimodal (200) fiber texture in BD and (111) fiber texture in TD to a significantly reduced preferred orientation, where only a weak impression of the (200) fiber texture remains. These changes occur between $\mathrm{n}=7$ and $\mathrm{n}=14$, with $100 \mu \mathrm{m}$ to $450 \mu \mathrm{m}$ of material between the gauge volume and the top layer exposed by the laser. After the total number of layers has reached $n=14$, no more intensity changes are observed.

The focused laser beam and its highly localized energy input, together with the extremely high heating and cooling rates, lead to lattice defects such as dislocations in LPBF [30]. The high defect density and the thermal cycling, combined with the fact that the material stayed in a solid state for the whole observation period, lead to the conclusion that DRX is the cause for the observed texture changes. While recovery, which is characterized by reducing lattice defects, results in a reduction in the FWHM, DRX leads to reformation of the microstructure, which results in the changes in the pole figures observed together with the reduction in the FWHM. These findings prove that the repeated laser scanning acts as an in situ heat treatment, which could be used to manipulate and tailor the resulting microstructure of the final part.

\section{In situ segregation stage}

In Sect. 3.3, a peak shape analysis was carried out. Anisotropic changes in the FWHM were observed.
An initial reduction in the FWHM coincided with texture changes, further supporting the DRX hypothesis discussed in the previous section. The peak width is influenced by several factors: temperature, domain size, microstrains, defect density.

Temperature can be excluded as a cause since it would impact the whole diffraction pattern, i.e., the whole azimuthal range equally. Domain size is inversely proportional to the peak width, and smaller domains lead to broader peaks. A decrease in domain size is possible during recrystallization, but after the initial microstructural changes, repeated laser scans are expected to lead to grain growth and, therefore, an increased domain size, resulting in a smaller FWHM. It is unlikely that there is a grain size reduction in $\mathrm{BD}$ at later stages in the process.

Defect density and microstrains are correlated. Defects such as stacking faults, dislocations, and subgrain boundaries exert a stress field, which leads to microstrains. In this case, the initial reduction in FWHM corresponds to the annihilation of lattice defects due to recovery and DRX. The increase in later stages could be attributed to an increase in microstrains. Due to the increased FWHM values in $\mathrm{BD}$, higher strains and defect concentrations are expected in this direction. As mentioned in the results section, the FWHM values appear to stay constant starting from $n=40$, implying that no new defects are generated from hereon.

Additionally, the peak asymmetry was evaluated. In an earlier study [20], this asymmetry was attributed to powder material irradiated simultaneously with the solidified material. However, the changes in asymmetry observed in the present study indicate that this is not actually the case. Instead, the increase in asymmetry implies a change in the phase composition of the material.

On one hand, Inconel 625 forms coherent precipitates after heat treatment [15]. Sarkar et al. [31] reported that these precipitates show diffracted peaks overlapping to the matrix. Especially the $\gamma^{\prime \prime}$ phase leads to a peak shape also found in the present study. It is difficult to extract the exact contribution of the precipitates, which is why their presence is often analyzed via changes of the matrix peak as described by Rai et al. [32]. The $\gamma^{\prime \prime}$ phase forms a small peak that contributes to the left-sided asymmetry presented in Sect. 3.3.

On the other hand, $\mathrm{Nb}$ and Mo segregation is a known phenomenon for Inconel 625 processed via 
LPBF [15], especially in the as-built state. Chemical inhomogeneities of any kind, including segregations, will impact the diffraction peak shape because they change the lattice spacing in the gauge volume. $\mathrm{Nb}$ and Mo-rich areas, found in the ex situ TEM investigations, have a different lattice spacing compared to regions with the nominal chemical composition, which will also induce an asymmetry in the diffraction peak.

Therefore, the increase in asymmetry that was observed indicates an increase in segregation concentration. The repeated laser scanning apparently promotes the growth of segregations in situ. This is an interesting and new finding since the impact of the laser on the gauge volume is expected to be reduced with an increasing number of layers. From these results, it appears that the energy input is high enough to promote the growth of segregations until the very end of the process, where there are more than $4.5 \mathrm{~mm}$ of material between the powder layer and the gauge volume. The TEM results clearly show $\mathrm{Nb}$ and Mo segregations, thereby supporting the hypothesis that the segregation growth is depicted by the in situ diffraction experiments.

\section{Conclusions}

This study shows that in situ diffraction experiments are a viable tool for texture analysis during laser powder bed fusion. Several factors impact the formation and evolution of texture. The energy input plays a significant role in the texture in the part. A combination of high laser power and scanning speed, but with a lower line energy $E_{\mathrm{L}}$, results in a stronger preferred orientation than low laser power and scanning speed with a higher line energy $E_{\mathrm{L}}$.

Additionally, the observation of a constant gauge volume revealed the impact of repeated laser scanning and the heat-affected zone on the microstructure. Significant texture changes were found during laser scanning up to a distance of ten layers below the working plane. The preferred orientation was strongly reduced. This texture change is attributed to recrystallization since the material stays in a solid state during this observation period. The present study is the first to experimentally observe this phenomenon in situ to the authors' knowledge. After the recrystallization stage, further microstructural changes occur. Apparently, lattice defects reappear after an initial reduction during recrystallization with a prominent preference for the building direction. This insight was gathered from an analysis of the diffraction peak FWHM. After a total number of 40 layers, no further changes of the FWHM occurred, but the peak shape still changed. Evaluating the peak asymmetry revealed an increase in an underlying contribution to the individual hkl reflections. This underlying contribution is related to the formation and growth of segregations, which was confirmed via ex situ TEM investigations. The peak asymmetry increases up to the end of the observation period at $\mathrm{n}=100$ layers, which underlines the impact of the repeated laser scanning during the segregation stage.

The results presented in this study contribute to the understanding of microstructure formation in LPBF. The in situ observations of recrystallization and segregation formation emphasize the complexity of thermal management during LPBF and highlight the relevance and capabilities of high-energy synchrotron radiation diffraction experiments to advance the fundamental process understanding. Further investigations are necessary to fully understand the influence of process parameters on the microstructure and facilitate a targeted microstructure design during the process.

\section{Acknowledgements}

The authors acknowledge the Deutsche Forschungsgemeinschaft (DFG) for funding research project No. 317078200. In addition, they acknowledge Helmholtz-Zentrum Hereon for the provision of laboratory facilities and support during the in situ diffraction experiments. Sören Selve and Christian Günther from Zentraleinrichtung Elektronenmikroskopie (ZELMI) and Anton Thiel from the department of Metallic Materials, all from Technische Universität Berlin, are acknowledged for conducting the STEM investigations and necessary sample preparation. The STEM device is funded by DFG (No. 403371556).

\section{Funding}

Open Access funding enabled and organized by Projekt DEAL. This study was funded by Deutsche Forschungsgemeinschaft (project No. 317078200). 


\section{Declarations}

Conflict of interest The authors declare that they have no conflict of interest.

Open Access This article is licensed under a Creative Commons Attribution 4.0 International License, which permits use, sharing, adaptation, distribution and reproduction in any medium or format, as long as you give appropriate credit to the original author(s) and the source, provide a link to the Creative Commons licence, and indicate if changes were made. The images or other third party material in this article are included in the article's Creative Commons licence, unless indicated otherwise in a credit line to the material. If material is not included in the article's Creative Commons licence and your intended use is not permitted by statutory regulation or exceeds the permitted use, you will need to obtain permission directly from the copyright holder. To view a copy of this licence, visit http://creativecommons.org/licen ses/by $/ 4.0 /$.

\section{References}

[1] Wassermann G, Grewen J (1962) Texturen metallischer Werkstoffe, 2nd edn. Springer, Berlin Heidelberg

[2] Wang Y, Yu C, Xing L, Li K, Chen J, Liu W, Ma J, Shen Z (2020) Grain structure and texture of the SLM single track.

J Mater Process Technol. https://doi.org/10.1016/j.jmatpro tec.2020.116591

[3] Wei HL, Elmer JW, DebRoy T (2016) Origin of grain orientation during solidification of an aluminum alloy. Acta Mater. https://doi.org/10.1016/j.actamat.2016.05.057

[4] Sun Z, Tan X, Tor SB, Chua CK (2018) Simultaneously enhanced strength and ductility for 3D-printed stainless steel 316L by selective laser melting. NPG Asia Mater. https://doi. org/10.1038/s41427-018-0018-5

[5] Yin J, Peng G, Chen C, Yang J, Zhu H, Ke L, Wang Z, Wang D, Ma M, Wang G, Zeng X (2018) Thermal behavior and grain growth orientation during selective laser melting of Ti6Al-4V alloy. J Mater Process Technol. https://doi.org/10. 1016/j.jmatprotec.2018.04.035

[6] Wang Di, Song C, Yang Y, Bai Y (2016) Investigation of crystal growth mechanism during selective laser melting and mechanical property characterization of 316L stainless steel parts. Mater Des. https://doi.org/10.1016/j.matdes.2016.03. 111

[7] Wang Z, Guan K, Gao M, Li X, Chen X, Zeng X (2012) The microstructure and mechanical properties of deposited-
IN718 by selective laser melting. J Alloy Compd. https://doi. org/10.1016/j.jallcom.2011.10.107

[8] Zhang D, Zhang P, Liu Z, Feng Z, Wang C, Guo Y (2018) Thermofluid field of molten pool and its effects during selective laser melting (SLM) of Inconel 718 alloy. Addit Manuf. https://doi.org/10.1016/j.addma.2018.03.031

[9] Gong H, Gu H, Zeng K, Dilip J, Pal D, Stucker B, Christiansen D, Beuth J, Lewandowski JJ (2014) In: Proceedings of the 25th Solid Freeform Fabrication Symposium, pp 256-267

[10] King WE, Barth HD, Castillo VM, Gallegos GF, Gibbs JW, Hahn DE, Kamath C, Rubenchik AM (2014) Observation of keyhole-mode laser melting in laser powder-bed fusion additive manufacturing. J Mater Process Technol. https://doi. org/10.1016/j.jmatprotec.2014.06.005

[11] Yap CY, Chua CK, Dong ZL, Liu ZH, Zhang DQ, Loh LE, Sing SL (2015) Review of selective laser melting: materials and applications. Appl Phys Rev. https://doi.org/10.1063/1. 4935926

[12] Roehling TT, Shi R, Khairallah SA, Roehling JD, Guss GM, McKeown JT, Matthews MJ (2020) Controlling grain nucleation and morphology by laser beam shaping in metal additive manufacturing. Mater Des. https://doi.org/10.1016/j. matdes.2020.109071

[13] Dehoff RR, Kirka MM, Sames WJ, Bilheux H, Tremsin AS, Lowe LE, Babu SS (2015) Site specific control of crystallographic grain orientation through electron beam additive manufacturing. Mater Sci Technol. https://doi.org/10.1179/ 1743284714Y.0000000734

[14] Sames WJ, List FA, Pannala S, Dehoff RR, Babu SS (2016) The metallurgy and processing science of metal additive manufacturing. Int Mater Rev. https://doi.org/10.1080/0950 6608.2015.1116649

[15] Marchese G, Lorusso M, Parizia S, Bassini E, Lee J-W, Calignano F, Manfredi D, Terner M, Hong H-U, Ugues D, Lombardi M, Biamino S (2018) Influence of heat treatments on microstructure evolution and mechanical properties of Inconel 625 processed by laser powder bed fusion. Mater Sci Eng A. https://doi.org/10.1016/j.msea.2018.05.044

[16] Sabzi HE, Aboulkhair NT, Liang X, Li X-H, Simonelli M, Fu H, Rivera-Díaz-del-Castillo PE (2020) Grain refinement in laser powder bed fusion: The influence of dynamic recrystallization and recovery. Mater Des. https://doi.org/10. 1016/j.matdes.2020.109181

[17] Hocine S, van Swygenhoven H, van Petegem S, Chang CST, Maimaitiyili T, Tinti G, Ferreira Sanchez D, Grolimund D, Casati N (2020) Operando X-ray diffraction during laser 3D printing. Mater Today. https://doi.org/10.1016/j.mattod.201 9.10 .001 
[18] Zhao C, Fezzaa K, Cunningham RW, Wen H, de Carlo F, Chen L, Rollett AD, Sun T (2017) Real-time monitoring of laser powder bed fusion process using high-speed X-ray imaging and diffraction. Sci Rep. https://doi.org/10.1038/s4 1598-017-03761-2

[19] Calta NP, Thampy V, Lee DR, Martin AA, Ganeriwala R, Wang J, Depond PJ, Roehling TT, Fong AY, Kiss AM, Tassone CJ, Stone KH, Nelson Weker J, Toney MF, van Buuren AW, Matthews MJ (2020) Cooling dynamics of two titanium alloys during laser powder bed fusion probed with in situ X-ray imaging and diffraction. Mater Des. https://doi. org/10.1016/j.matdes.2020.108987

[20] Schmeiser F, Krohmer E, Schell N, Uhlmann E, Reimers W (2020) Experimental observation of stress formation during selective laser melting using in situ X-ray diffraction. Addit Manuf. https://doi.org/10.1016/j.addma.2019.101028

[21] Schmeiser F, Krohmer E, Schell N, Uhlmann E, Reimers W (2021) Internal Stress evolution and subsurface phase transformation in titanium parts manufactured by laser powder bed fusion - an in situ x-ray diffraction study. Adv Eng Mater. https://doi.org/10.1002/adem.202001502

[22] Wahlmann B, Krohmer E, Breuning C, Schell N, Staron P, Uhlmann E, Körner C (2021) In situ observation of $\gamma^{\prime}$ phase transformation dynamics during selective laser melting of CMSX-4. Adv Eng Mater. https://doi.org/10.1002/adem. 202100112

[23] Uhlmann E, Krohmer E, Schmeiser F, Schell N, Reimers W (2020) A laser powder bed fusion system for in situ X-ray diffraction with high-energy synchrotron radiation. Rev Sci Instrum. https://doi.org/10.1063/1.5143766

[24] Schell N, King A, Beckmann F, Fischer T, Müller M, Schreyer A (2013) The high energy materials science beamline (HEMS) at PETRA III. MSF. https://doi.org/10. 4028/www.scientific.net/MSF.772.57

[25] Ashiotis G, Deschildre A, Nawaz Z, Wright JP, Karkoulis D, Picca FE, Kieffer J (2015) The fast azimuthal integration
Python library: pyFAI. J Appl Crystallogr. https://doi.org/10. 1107/S1600576715004306

[26] Bachmann F, Hielscher R, Schaeben H (2010) Texture analysis with MTEX - free and open source software toolbox. SSP. https://doi.org/10.4028/www.scientific.net/SSP.16 0.63

[27] Newville M, Stensitzki T, Allen DB, Ingargiola A (2014) LMFIT: Non-Linear Least-Square Minimization and CurveFitting for Python. Zenodo

[28] Wenk H-R, Grigull S (2003) Synchrotron texture analysis with area detectors. J Appl Crystallogr. https://doi.org/10. 1107/S0021889803010136

[29] Bidare P, Maier R, Beck RJ, Shephard JD, Moore AJ (2017) An open-architecture metal powder bed fusion system for insitu process measurements. Addit Manuf. https://doi.org/10. 1016/j.addma.2017.06.007

[30] Small KA, Clayburn Z, DeMott R, Primig S, Fullwood D, Taheri ML (2020) Interplay of dislocation substructure and elastic strain evolution in additively manufactured Inconel 625. Mater Sci Eng A. https://doi.org/10.1016/j.msea.2020. 139380

[31] Sarkar A, Mukherjee P, Barat P, Jayakumar T, Mahadevan S, Rai SK (2008) Lattice misfit measurement in Inconel 625 by X-ray diffraction technique. Int J Mod Phys B:3977-3985. h ttps://doi.org/10.1142/s0217979208048772

[32] Rai SK, Kumar A, Shankar V, Jayakumar T, Bhanu Sankara Rao K, Raj B (2004) Characterization of microstructures in Inconel 625 using X-ray diffraction peak broadening and lattice parameter measurements. Scr Mater. https://doi.org/ 10.1016/j.scriptamat.2004.03.017

Publisher's Note Springer Nature remains neutral with regard to jurisdictional claims in published maps and institutional affiliations. 